\title{
Aproximaciones didácticas en el proceso educativo
}

\author{
Edith Judith Grosso Asprilla * \\ James Rosas Villanueva **: \\ Nelson Augusto Medina Peña ${ }^{* * *}$
}

Artículo de reflexión.

Fecha de Recepción: 23 julio 2018.

Fecha de Aprobación: 29 octubre 2018

\section{Resumen}

Con el presente artículo, se busca dar a conocer el desarrollo parcial de la investigación intitulada "El juego en química y matemáticas, alternativa para la apatía en su aprendizaje”, adelantada en la Institución Educativa Mariano Ospina Pérez del municipio de Tinjacá, donde luego de un seguimiento a las clases de estas dos áreas se evidenció un sentir de falta de interés y desagrado por su estudio; por lo que se hizo necesario implementar un proyecto que relacionara estas áreas y cómo debería darse su enseñanza, implícito en la relación pedagógica en las instituciones educativas, para así buscar mejorar la actitud que los estudiantes tenían hacia estas. Esto a partir de acciones como charlas e interacción con los estudiantes, donde se marcó que el juego era una buena alternativa; por lo que, se procedió a crear e implementar el juego dentro del desarrollo de las clases. Así, se pudieron ver avances en la forma en que los estudiantes se desempeñaban, pues, hacerlos partícipes del proyecto y creadores de su propio material didáctico dejó ver motivación $y$, sobre todo, compromiso con su propia formación, lo que nos reveló que vamos por buen camino porque los aportes de esta investigación han mostrado cambios bastante marcados y, sobre todo, positivos, aunque ha pasado poco tiempo de su aplicación.

Palabras clave: apatía, relación pedagógica, juego, química, matemáticas.
* Institución Educativa Mariano Ospina Pérez, Boyacá (Colombia) edjugroas60@gmail.com ** Institución Educativa Mariano Ospina Pérez, Boyacá (Colombia) jarovi696@yahoo.es *** Universidad Pedagógica y Tecnológica de Colombia, Boyacá (Colombia) nelsonaugusto.medina@ptc. edu.co

(c)(9) $\Theta$ 


\section{Introducción}

A través del tiempo, se ha venido percibiendo una gran apatía en los estudiantes de secundaria hacia el estudio de la química; y desde la primaria, al estudio de las matemáticas. Así lo confirma el estudio realizado por Valverde y Naslund-Hadleu (2010), quienes evidenciaron esta condición de la enseñanza de estas dos áreas a través de un informe que recoge las experiencias en diferentes países de América Latina y el Caribe.

Los jóvenes de hoy se han escudado en las reformas educativas para convertirse en estudiantes poco competitivos, perezosos, apáticos al aprendizaje, y la relación pedagógica distante con sus docentes acentúa esta situación; esta era la percepción que existía en el ambiente escolar de la Institución Educativa Mariano Ospina Pérez del municipio de Tinjacá, motivo por el cual se planteó la pregunta ¿Cómo establecer una relación pedagógica con los estudiantes de grado $9^{\circ}$, para disminuir la apatía y mejorar los ambientes escolares en el aprendizaje de las áreas de Química y Matemáticas?, como punto de partida para la presente investigación.

Teniendo en cuenta lo enunciado, se planteó como propósito general de la investigación, establecer una relación pedagógica con los estudiantes de grado noveno de la Institución Educativa Mariano Ospina Pérez del municipio de Tinjacá, para disminuir la apatía y mejorar los ambientes escolares en el aprendizaje de las áreas de Química y Matemáticas, esto a partir del desarrollo de los objetivos específicos, que fueron: analizar las posibles causas que generan apatía para aprender química y matemáticas en estudiantes del grado noveno de la Institución Educativa, desarrollar una propuesta pedagógica que contribuya a fortalecer el aprendizaje de química y matemáticas, y describir los aportes de la propuesta pedagógica desarrollada.

Finalmente, es importante dejar claro que en el presente documento se consolida información correspondiente a la investigación intitulada " $\mathrm{El}$ juego en química y matemáticas, alternativa a la apatía en su aprendizaje", la cual hace parte del desarrollo de estudios de la Maestría en Educación, en el marco del programa de becas para la excelencia del Ministerio de Educación Nacional. Esta investigación está en curso, por ende no se presentan resultados finales.

\section{Aportes teóricos}

A partir de la contextualización del tema, se abordan aportes realizados en investigaciones tanto a nivel internacional como nacional, cuyos resultados respaldan la investigación adelantada en esta institución, al igual que estudios propios de los ejes temáticos que están directamente relacionados.

\section{Relación pedagógica}

Al hablar de relación pedagógica, primero que todo debemos romper con el paradigma que nos lleva una relación asimétrica de poderes, donde el profesor tiene un estatus superior 
al del estudiante, pues de esta manera no se podría establecer dicha relación de manera efectiva. La comunicación entre estudiantes y docentes, debe darse al mismo nivel para que esta fluya de la mejor manera. Hace varios años, muchas instituciones educativas tenían una zona en sus aulas que era más alta que el resto del aula, cuyo lugar era usado para que el profesor impartiera desde allí su clase, viéndose de esta manera con un grado de superioridad con respecto a sus alumnos, pero con el paso del tiempo este espacio fue demolido justamente con el fin de hacer ver al docente al mismo nivel que el de sus estudiantes.

Así mismo, según Contreras (citado en Paredes, 2011), la relación pedagógica establece un vínculo entre docentes y estudiantes en su proceso de formación en las Instituciones educativas. Esta relación está siempre presente (desde que el estudiante llega al aula hasta que el aula queda vacía; en las actitudes, diálogo, en la interacción entre docentes y estudiantes, entre otras) y es muy importante tenerla en cuenta, pues es desde esta que se busca una adecuada comunicación entre los participantes del proceso enseñanza-aprendizaje en las aulas de clase, para lograr los objetivos educativos que inicialmente se plantean, esto, teniendo en cuenta que, según Barba (citado en Paredes, 2011) "El alumno es considerado un sujeto constructor de su propio conocimiento y aprendizaje, por lo cual es necesario fomentarle un ambiente de libertad y autonomía que propicie su participación activa y continua en el proceso de enseñanza-aprendizaje"(p.
2), sin desconocer el papel del docente como guía en el proceso de educación.

La relación pedagógica debe ir en doble vía, es decir, que ambos actores (tanto docentes como estudiantes) deben tener un aprendizaje continuo, porque es claro que el docente no es el único que enseña y quien sabe, ni el estudiante el único en disposición de aprender en medio de este proceso, lo que convierte al docente en acompañante, guía, orientador y quien propone actividades que faciliten el aprendizaje, siempre dispuesto a recibir aportes de sus estudiantes, enriqueciendo de esta manera dicha relación.

\section{Apatía}

Etimológicamente, el término "apatía" se deriva del latín apathia, y este del griego, que significa ausencia de pasiones, emociones, sentimientos o enfermedad. La apatía puede definirse como la impasibilidad del ánimo y dejadez, indolencia, falta de vigor o energía. También, podría decirse que es un estado de desinterés acompañado de la falta de expresión de emociones, tales como la preocupación, exaltación o pasión por lo que está pasando o viviéndose en una situación determinada.

La apatía puede presentarse por diferentes causas y en diferentes momentos o etapas de la vida, el desinterés por diferentes actividades en la etapa de la preadolescencia y adolescencia, marca considerablemente la vida de un ser humano, teniendo en cuenta que es en este momento donde se inicia su vida académica y se
Contreras (citado en Paredes, 2011), la relación pedagógica establece un vínculo entre docentes y estudiantes en su proceso de formación en las Instituciones educativas. 
Desde la mirada de Piaget, (citado en Teoría del juego, 2012), "El juego forma parte de la inteligencia del niño, porque representa la asimilación funcional o reproductiva de la realidad según cada etapa evolutiva del individuo" toman decisiones importantes acerca de su futuro educativo. En la escuela surge cuando el alumno se encuentra desconectado de la clase. Usualmente distraído o desinteresado, simplemente atiende a la materia, pero en realidad, su mente se encuentra en otro lado. El estado emocional que acompaña a la apatía es variable, puede ser desde el estudiante aburrido, hiperactivo o agresivo.

En el caso particular de la química, Rodríguez (2013) hace referencia al tema en su artículo llamado "El aprendizaje de la Química de la vida cotidiana en la Educación Básica"; y para las matemáticas, Vacca (2012), en el estudio llamado "Apatía hacia las matemáticas"

\section{Ambientes de aprendizaje}

Por otro lado, teniendo en cuenta que uno de los propósitos de esta investigación es mejorar los ambientes de aprendizaje en el área de química y matemáticas, es necesario tener en cuenta que, para buscar un ambiente ideal que favorezca la relación pedagógica de tal manera que sirva como medio para lograr un aprendizaje significativo en el estudiante, se debe partir desde el conocimiento y reconocimiento de su contexto, pues sin esto sería poco pertinente intentar cualquier acción dentro del aula, pues este influye en el desarrollo que tiene cada estudiante en las diferentes áreas del conocimiento.

De acuerdo con organizaciones como la UNESCO, un ambiente escolar acogedor, respetuoso y positivo, es una de las claves para promover el aprendizaje de los estudiantes y la obtención de altos logros en las pruebas externas. También, puede destacarse que las condiciones al interior de la escuela influencian el desempeño de los estudiantes, favoreciendo significativamente la disminución de las desigualdades de aprendizaje asociadas a las disparidades económicas y sociales de ellos.

\section{El juego didáctico}

El juego es una práctica que es innata en el ser humano, porque este presenta sus primeras manifestaciones cuando juega con sus manos o sus pies en los primeros meses de vida, como un proceso de aprendizaje y reconocimiento propio. El juego se potencializa en los primeros años de vida y aún más cuando empieza la etapa escolar con la cual el niño crea lazos de amistad y emplea, a través de este, un nuevo lenguaje de comunicación. Según Gross (citado en Teoría del juego, 2012), "Es objeto de una investigación psicológica especial, siendo el primero en constatar el papel del juego como fenómeno de desarrollo del pensamiento y de la actividad" (párr. 1). Desde la mirada de Piaget, (citado en Teoría del juego, 2012), "El juego forma parte de la inteligencia del niño, porque representa la asimilación funcional o reproductiva de la realidad según cada etapa evolutiva del individuo"(parr.5). Según Vygotsky (citado en Teoría del juego, 2012), "El juego surge como necesidad de reproducir el contacto con los demás. Naturaleza, origen y fondo del juego son fenómenos de tipo social, y a través 
del juego se presentan escenas que van más allá de los instintos y pulsaciones internas individuales" (parr.15).

Por otro lado, a medida que el niño crece, el juego se alimenta con la participación en los diferentes roles que le permiten fomentar su liderazgo e incrementar su vocabulario. Sin embargo, a medida que los niños crecen el juego se convierte tan solo en una práctica para realizar únicamente en momentos de descanso en las Instituciones Educativas luego de haber cumplido con las actividades programadas por el docente, aunque "El juego didáctico es una estrategia que se puede utilizar en cualquier nivel o modalidad del proceso educativo, pero por lo general el docente lo utiliza muy poco porque desconoce sus múltiples ventajas. El juego que posee un objetivo educativo, se estructura como un juego reglado que incluye momentos de acción reflexiva y de simbolización o apropiación" (Chacón, 2008. Parr.1). La combinación del juego y las actividades académicas, es una mezcla perfecta, teniendo en cuenta que los niños aprenden sin presión $y$, por el contrario, se puede realizar con la relajación propia de un juego, los niños pueden participar con espontaneidad, sin el temor de ser señalado o corregido con rigidez como suele suceder en una clase tradicional (Gómez-Duarte \& Peñaloza-Jiménez, 2014).

Esta práctica se puede realizar también con los estudiantes de grados superiores, quienes ven el juego como una actividad completamente desligada de su quehacer como estudiantes. Con ellos, además de abarcar los temas propios de las asignaturas, se puede fortalecer las relaciones interpersonales, el respeto por el otro, la competencia sana y recuperar la importancia que se ha perdido del juego, convierte a los estudiantes en estrategas para tener buenos resultados en su participación, los conduce a pensar en diversas formas para resolver un problema y a expresar distintas formas de pensamiento. "El uso de los juegos en la educación matemática es una estrategia que permite adquirir competencias de una manera divertida y atractiva para los alumnos" (Muñiz, Alonso \& Rodríguez, 2014, p. 19).

El juego hace parte de diferentes disciplinas deportivas en las que se destaca el contacto físico directo, sin embargo, "El juego bueno, el que no depende de la fuerza o maña físicas, el juego que tiene bien definidas sus reglas y que posee cierta riqueza de movimientos, suele prestarse muy frecuentemente a un tipo de análisis intelectual cuyas características son muy semejantes a las que presenta el desarrollo matemático" (De Guzmán, 1984, párr. 2). Por otro lado, Fernández (2014) asegura que, "gracias a la utilización de los juegos, podemos cambiar la idea de que las matemáticas son aburridas, tan generalizado hoy en día” (p. 59).

Respecto a la enseñanza de la química, un estudio llamado "Didáctica de la química a través de los juegos" publicado por la Revista digital para profesionales de la enseñanza (2010),
"El juego didáctico es una estrategia que se puede utilizar en cualquier nivel o modalidad del proceso educativo, pero por lo general el docente lo utiliza muy poco porque desconoce sus múltiples ventajas. El juego que posee un objetivo educativo, se estructura como un juego reglado que incluye momentos de acción reflexiva y de simbolización o apropiación" 
hace referencia a que los "juegos didácticos ofrecen la posibilidad de un trabajo cooperativo y potencia que los alumnos sean gestores de su propia formación y desarrollo" (p. 9). Por otro lado, Guapacha (2013), en su estudio llamado; El juego como estrategia en la enseñanza-aprendizaje de la Nomenclatura Inorgánica, evidencia que "Implementar juegos para la Enseñanza-Aprendizaje de la Nomenclatura Inorgánica, es viable puesto que posibilita no solo la apropiación de los conceptos básicos, sino que cambia la idea de que la clase de química es aburrida y que existen otras maneras de aprender y enseñar"(p.89).

Cabe anotar que, cuando hablamos del juego, este se toma como una actividad placentera para quien la práctica. Por lo que, puede llegar a confundirse con la lúdica, por lo tanto, es necesario hacer una diferenciación entre estos dos conceptos.

\section{La lúdica}

Puede decirse que es una forma grata de ver, sentir y vivir las diferentes situaciones de la vida y, en especial, en educación es aquella que permite un tranquilo transcurrir de la vida escolar, agradable y satisfactoria. Desde aquí, hacer intervenciones sobre los diferentes procesos en los estudiantes en su desarrollo cognitivo durante su tiempo de aprendizaje.

Según Posada (2014), “La lúdica se toma como una forma de ser, una manera de interactuar con diversas facetas, para hacerlas más manejables en la incertidumbre de la realidad, característica esencial de la vida" (p. 11). Donde el juego toma parte importante, mas no es exclusivamente lo que compete al accionar lúdico, sino una de sus componentes más importantes.

La lúdica en el aprendizaje surge como aporte a la posibilidad de producir cambios positivos, un espacio de construcción cultural, social y de conocimiento (Samacá, 2016).

\section{Metodología}

El proceso metodológico establecido para el desarrollo de la investigación, parte de una realidad como lo es la apatía al aprendizaje, aspecto que se viene presentando en procesos educativos a nivel general. Se fundamenta en un enfoque cualitativo, que, según Rodríguez, Gil y García (1996), "estudia la realidad en su contexto natural, tal y como sucede, intentando sacar sentido de, o interpretar los fenómenos de acuerdo con los significados que tiene para las personas implicadas" (p. 32), y bajo el tipo de investigación acción educativa, la cual resulta ser una invitación para que los docentes revisen, reorganicen y reflexionen sobre su práctica educativa, con el fin de "optimizar los procesos de enseñanzaaprendizaje." (Elliot, citado en Bausela, 2002). De esta manera, la investigación se ve encaminada a fortalecer la praxis pedagógica permitiendo, a su vez, mayor participación de los estudiantes, la interacción entre ellos y la dinamización del proceso educativo. En este sentido, la investigación permite realizar una transformación 
en la educación, de tal manera que esta se vea reflejada, a su vez, en la sociedad (Orrego \& Toro, 2014); en este proceso, los estudiantes serán miembros activos, ya que el trabajo no puede desligar ninguna de estas dos partes.

A partir de los anteriores elementos, se planteó una ruta metodológica estructurada en tres fases con sus respectivas etapas, así:

Fase de Diagnóstico, consistió en el desarrollo de unas etapas, las cuales en su conjunto fortalecieron el proceso y permitieron avanzar en el logro de los objetivos, estas fueron: etapa de observación directa, que consistió en visualizar las diferentes situaciones durante cada uno de los encuentros en clase y registradas en el diario de campo; la siguiente etapa que consistió en la revisión de documentos institucionales como: PEI (Proyecto Educativo Institucional), PMI (Plan de Mejoramiento Institucional, ISCE (Índice Sintético de Calidad Educativa); y la última etapa que consistió en la exploración, a través de la cual se elaboró un instrumento (entrevista), con el cual se buscaba analizar las posibles causas que generan apatía para aprender química y matemáticas.

Fase de planeación-acción, el desarrollo de esta fase se llevó a cabo a partir de los resultados obtenidos en la fase anterior, para lo cual se plantearon las siguientes etapas: etapa de exploración y concertación, que consistió en el diálogo e interacción entre estudiantes e investigadores, buscando proponer acciones que dieran razón al logro de una segunda etapa de esta fase, donde se decidió que fuera el juego aquella propuesta que se diera como mediador del conocimiento en el aula, dado que por naturaleza es muy llamativo y agradable. Además, se pensó en juegos conocidos en la región, para que fuese fácil su manejo y, luego, dar paso a una segunda, denominada Elaboración, que correspondió a la estructuración y elaboración de las diferentes acciones a adelantar teniendo en cuenta lo socializado y consolidado en la etapa anterior, que para el caso de este proyecto fue la elaboración de diferentes juegos (parques, bingo, entre otros) y adaptarlos al desarrollo de las diferentes temáticas de las áreas de química y matemáticas, para lo cual se formaron grupos de trabajo teniendo en cuenta las diferentes aptitudes de los estudiantes, lo cual fue gran ventaja para lograr el mejor material posible; y la última etapa de esta fase se denominó Desarrollo Práctico, esta etapa consistió en desarrollar las acciones determinadas y elaboradas, al proceso de enseñanza- aprendizaje desde cada una de las dos áreas. Donde básicamente se practicó con los juegos y se puso en marcha la propuesta de manera física en cada una de las sesiones de clase.

Fase de observación y reflexión. Este proceso se realizó de manera permanente desde el comienzo de la investigación, permitiendo llevar una secuencia de los avances durante la evolución del desarrollo. Cabe anotar que esta fase se ha venido desarrollando a través de todo el proceso investigativo, y que se sistematizó en diarios de campo, los cuales fueron el instrumento usado 
"históricamente se ha evidenciado una gran apatía en los estudiantes de bachillerato hacia el estudio de la química, esto puede observarse en el bajo rendimiento generalizado en esta asignatura, la escasa participación en los eventos científicos y hasta el manifiesto rechazo hacia la misma por los investigadores durante toda la investigación. Esta se ha venido llevado a cabo en la Institución Educativa Mariano Ospina Pérez del municipio de Tinjacá, la cual está dirigida actualmente por el especialista Jairo Vicente Cifuentes. Esta Institución se encuentra ubicada en el centro del municipio, cuenta con 10 sedes y aproximadamente 485 estudiantes, de los cuales un $80 \%$ pertenecen al sector rural; posee 27 docentes y 2 empleados que corresponden al área administrativa. La Institución es de carácter oficial, adscrita a la Secretaría de Educación de Boyacá. La unidad de análisis con la cual se adelantó la investigación, correspondió a estudiantes del grado $9^{\circ}$ que, para el presente año, fueron 47, de los cuales 21 son de género femenino y 26 de género masculino, cuyas edades oscilan entre 13 y 16 años.

Así mismo, se informa que el desarrollo de la investigación se ha venido realizando teniendo en cuenta las consideraciones éticas que competen como tal a este tipo de procesos.

\section{Resultados y discusión}

Como consecuencia de la aplicación de las fases anteriormente descritas, se han consolidado resultados de la primera fase (Fase de Diagnóstico), de la segunda (Planeación y acción) y de la tercera (Observación y reflexión) en forma parcial, así:

Fase de Diagnóstico, donde se determinó que la investigación responde a situaciones encontradas con el desarrollo de las respectivas etapas a saber, se dio inicio con la etapa de Observación directa, la cual se desarrolló a partir del mes de agosto del 2016 a los estudiantes que en su momento cursaban grado octavo. El resultado de dicha observación durante cada una de las clases de química y matemáticas, fue registrado en el diario de campo, en el que se evidencian los aspectos más relevantes $\mathrm{y}$, sobre todo, recurrentes del desempeño de los estudiantes y de su actitud frente al desarrollo de las áreas anteriormente relacionadas, estos resultados evidenciaron la presencia de apatía en el aprendizaje de muchos de los estudiantes que hacían parte de este grado.

Los aspectos descritos se venían dando como consecuencia de la relación de los estudiantes y el docente dentro del aula, y por la forma como se abordaban los ejes temáticos de química $\mathrm{y}$ matemáticas, tal como lo confirma en química el estudio de Rodríguez (2013), donde enuncia que "históricamente se ha evidenciado una gran apatía en los estudiantes de bachillerato hacia el estudio de la química, esto puede observarse en el bajo rendimiento generalizado en esta asignatura, la escasa participación en los eventos científicos y hasta el manifiesto rechazo hacia la misma” (p. 364); y en el caso puntual de las matemáticas, Vacca (2012) confirma que la apatía por el aprendizaje de esta área se da desde los niños que ingresan a sus primeros años de estudio, y que este persiste durante el resto de su vida escolar debido a que únicamente se ven limitados a imitar ejercicios y operaciones de 
manera mecánica, copiando el modelo enseñado por el profesor en el tablero, además de adoptar los planteamientos y soluciones de situaciones, tal y como lo presentan los textos escolares, sin tener en cuenta el contexto real del estudiante. Los estudios realizados por Rodríguez y Vacca, son una muestra de que la apatía abarca diferentes contextos educacionales y que el de la Institución Educativa Mariano Ospina Pérez no es ajeno a este fenómeno entre los estudiantes de grado noveno, lo que motivó a la realización de la presente investigación.

La segunda etapa, que correspondió a la revisión de documentos institucionales, permitió identificar aspectos importantes frente a las principales causas para que los estudiantes sientan apatía por el aprendizaje de las áreas de química y matemáticas, tal es el caso del ISCE (Índice Sintético de Calidad Educativa), donde se destaca el bajo puntaje en el componente de ambiente de aula, evaluado por los mismos estudiantes, según informe del 2015. Otro de los documentos institucionales revisados fue el PMI (Plan de Mejoramiento Institucional), donde se encuentra registrado en la Gestión académica en el apartado de prácticas pedagógicas, la necesidad de "establecer las opciones didácticas para las áreas, asignaturas y proyectos transversales", con el fin de que este esté enfocado hacia la innovación e institucionalización de aquellas metodologías que arrojan los mejores resultados.

Así, mismo, en el PMI (Plan de Mejoramiento Institucional), se tiene contemplado "la necesidad de que los docentes adopten nuevas estrategias para acercarnos a nuestros estudiantes y lograr con ellos una relación más cercana, más efectiva y así lograr mayor interés y atención en cada una de las clases en la institución educativa”. Lo anterior apoyado también el PEI (Proyecto Educativo Institucional), que presentó una reestructuración en el modelo pedagógico de la Institución a modelo Dialogante o interestructural, cuyo punto de partida son diferentes modelos pedagógicos que se complementan entre sí, como el constructivista, conductista, entre otros, que facilitan el logro las metas propuestas en el PMI (Plan de Mejoramiento Institucional) y que, a su vez, apoyan cabalmente esta investigación.

La tercera etapa fue la de exploración, que correspondió al diseño y aplicación de una entrevista informal a todos los estudiantes de grado noveno. La entrevista fue utilizada como instrumento para recoger la información de manera más concreta, de tal manera que pudiera corroborarse lo observado en la primera etapa de esta fase. Esta presentó resultados significativos que permiten ver el alcance del objetivo de la investigación, ya que a través de este instrumento, se logró identificar claramente cuáles eran las causas más relevantes que generaban apatía en los estudiantes en el aprendizaje de la química y las matemáticas.

También, se recibieron aportes importantes en cuanto a las estrategias 


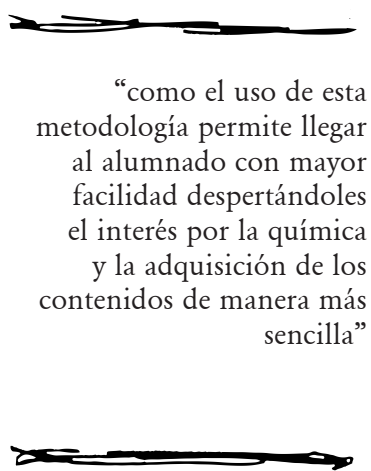

que, según los estudiantes, contribuirían a disminuir o abatir la apatía y que los harían sentir más cómodos durante el desarrollo de las clases en las dos áreas, estableciendo a su vez una relación más cercana, de confianza y agrado entre docentes y estudiantes a través del desarrollo de actividades, donde principalmente se destacó el juego, que en el área de matemáticas lo refiere Fernández (2014) en su estudio ”El juego y las matemáticas", "Los niños tienen que participar en clase, dar su opinión, experimentar cosas nuevas, sensaciones, ponerles retos, metas a alcanzar, donde una de las mejores maneras para que realicen todo esto es a través del juego"(p.59). Los resultados positivos de esta práctica también se encuentran relacionados en el área de química, a través del estudio llamado "Didáctica de la química a través de los juegos" publicado por la revista Digital para profesionales de la enseñanza (2010), donde se comprobó “como el uso de esta metodología permite llegar al alumnado con mayor facilidad despertándoles el interés por la química y la adquisición de los contenidos de manera más sencilla” (p. 9).

En la segunda fase (Planeación y acción), teniendo en cuenta los resultados de la última etapa de la fase anterior, se procedió a diseñar y aplicar una propuesta pedagógica de acuerdo con las siguientes etapas:

La primera etapa consistió en la exploración y concertación. Esta se adelantó en conjunto con los estudiantes para relacionar algunos tipos de juegos reconocidos en el contexto, de tal forma que pudiesen ser empleados y adaptados de manera didáctica, con el fin de aprovechar la familiaridad con estos para ser abordados en el desarrollo de los ejes temáticos de las áreas en mención, partiendo de que los juegos son viables en educación y "se pueden adaptar a cualquier contenido y son muy útiles para captar la atención del alumnado, hacer que comprendan mejor los conceptos, desarrollar habilidades y destrezas reforzar una actitud positiva ante la asignatura" (Fernández, 2014, p. $1)$.

De la misma manera, Guapacha (2013) afirma que se puede "cambiar la visión que se tiene acerca del juego que solo es útil en áreas como la educación física $y$ en niveles de primaria, por una que hacer ver la gran utilidad que el juego tiene en áreas como la química" (p. 88). Tomando como base la estrategia de jugar en algunas de las clases, los acuerdos continuaron dándose ahora para la elaboración de los juegos. Allí, se determinó qué juegos se construirían y cómo se harían, recomendando traer materiales que ya no se usaban en las casas o reciclarlos, lo cual sería importante para minimizar los costos, aunque cabe anotar que algunos materiales debían comprarse, pues se pretendía que los juegos fueran resistentes y de buena presentación.

La segunda etapa fue la elaboración de los juegos. Para su desarrollo, se destinaron los espacios durante el transcurso de algunas clases, durante el mes de noviembre del año 2016. Se organizaron grupos de tres estudiantes para trabajar teniendo en cuenta 
las diversas habilidades (diseño, dibujo, pintura) con las que cuentan los estudiantes de grado noveno, fortaleciéndose de esta manera el trabajo cooperativo en el que tuvieron la oportunidad de mostrar su liderazgo, el respeto por las opiniones de sus compañeros, el mejoramiento de sus relaciones interpersonales, entre otras.

Para la construcción de los juegos, los estudiantes recolectaron aquellos materiales recuperables de sus hogares encaminado a "La adquisición de una conciencia para la conservación, protección y mejoramiento del medio ambiente, de la calidad de la vida, del uso racional de los recursos naturales, de la prevención de desastres, dentro de una cultura ecológica y del riesgo y de la defensa del patrimonio cultural de la nación”. Como está establecido en la Ley general de educación Artículo $5^{\circ}$. Fue necesario tener en cuenta que, algunas de las acciones como cortar y pulir madera no eran posibles de realizar en la institución educativa, debido a que no se contaba con las herramientas para tal propósito, motivo por el cual se hizo necesario llevar material para realizarlas en sus hogares o en lugares apropiados para tal fin. Ya construido cada juego, se procedió a guardarlo en estantes, en el aula de cada área, con el fin de conservarlo en las mejores condiciones físicas y que siempre se tuviera fácil acceso a ellos.

La tercera etapa correspondió al desarrollo práctico, las reglas con las que se practican tradicionalmente los juegos fueron ligeramente modificadas haciendo énfasis en el propósito de ser usadas para facilitar el aprendizaje de los ejes temáticos de las áreas de química y matemáticas. Los juegos adoptados en la etapa anterior y usados para desarrollar la propuesta, fueron: parqués, escalera, dominó, bingo, lotería y concéntrese; algunos juegos fueron diseñados de tal manera que pudieran servir para diferentes temáticas. Antes de empezar a jugar, fue necesario puntualizar las reglas para cada uno de los juegos; $y$, en algunos casos, fue necesario enseñar a jugar porque a pesar que son juegos cotidianos y del entorno de los estudiantes, hubo pocas excepciones en las que los estudiantes jamás habían practicado alguno de los juegos por ellos elegidos para aplicar la propuesta.

El desarrollo de este proceso permitió potencializar el trabajo cooperativo, ya que los estudiantes siempre trabajan en grupo, intercambian de compañeros y de juego, permitiendo tener aportes significativos de todos sus compañeros y fortaleciendo, a su vez, las relaciones interpersonales con ellos y con el docente, creando así una relación pedagógica con mayor fluidez, teniendo en cuenta que el docente es un participante activo durante todo el proceso, tal y como lo refiere Contreras (citado en Paredes, 2011) "la relación pedagógica, se refiere al acto que ocurre en las aulas y escuelas entre profesores y estudiantes, lo cual parece es el sentido original de la profesión docente, estar con los jóvenes y poder orientarles" (párr. 1), lo cual se dio durante cada uno de los encuentros, durante el desarrollo de las clases en las áreas mencionadas anteriormente.

"La adquisición de
una conciencia para la
conservación, protección
y mejoramiento del medio
ambiente, de la calidad de
la vida, del uso racional de
los recursos naturales, de
la prevención de desastres,
dentro de una cultura
ecológica y del riesgo y de
la defensa del patrimonio
cultural de la nación."


En la tercera fase (Observación $\mathbf{y}$ reflexión), se continuó usando el diario de campo como instrumento para seguir registrando cada uno de los avances del desarrollo de la investigación, de la misma manera que se hizo durante cada una de las etapas anteriores. Durante el desarrollo de esta fase, que se encuentra aún en construcción, se ha venido registrando la incidencia que ha tenido el juego usado como estrategia didáctica durante el aprendizaje de la química y las matemáticas. También, se identificó el cambio de actitud durante el abordaje de las mismas, la disminución de la falta de interés que dio origen a esta investigación, donde Guapacha (2013), concluye que "El juego es una estrategia que motiva y despierta mayor interés en los estudiantes en el aprendizaje de la Nomenclatura Inorgánica"(p.88); $\mathrm{y}$ en las matemáticas, tal como se corrobora con el estudio realizado por Muñiz, Alonso \& Rodríguez (2014), donde "A lo largo de las distintas sesiones, por medio de la observación en el aula, se percibió un aumento de la motivación en los alumnos. La mayoría de los discentes se implicaban en las actividades, mostrándose participativos"(p.12). También se han venido obteniendo mejores resultados académicos en pruebas internas (registrados planilla de calificaciones de cada una de las áreas relacionadas).

\section{Consideraciones parciales}

Reflexionar continuamente sobre las prácticas educativas por parte de cada uno de los docentes en cualquier institución, es sin duda el primer paso que debe darse para buscar mejorar cualquier situación que pueda presentarse al interior de sus aulas, pues es en este proceso donde se puede detectar qué pasa con los estudiantes y por qué se da una situación determinada. Que, para el caso de esta investigación, nos muestra que la apatía en las clases es un factor determinante en su desarrollo y que no solo se convierte en obstáculo entre docente y estudiantes, sino también en un agente desmotivador que trae consecuencias, como: desinterés por el estudio y desagrado en la permanencia en las aula.

Fortalecer la relación pedagógica dentro del proceso educativo, es esencial para mantener una buena actitud frente al desarrollo de cualquier clase en el aula, ya que esta implica el agrado y la sensación de bienestar por parte del estudiante y un vínculo más cercano con sus docentes durante el desarrollo de las clases, en la búsqueda por mantener el interés por el aprendizaje de las áreas de química y matemáticas durante cada una de las sesiones. Por lo que, el juego se convirtió en importante vínculo entre los docentes y sus estudiantes, pues, los acercó más, los estudiantes se sintieron más a gusto durante las clases y no solo se sintieron como receptores de un conocimiento y estrategias de aprendizaje ya determinadas.

Usar el juego en la relación pedagógica ha determinado un cambio sustancial en la actitud de los estudiantes Institución Educativa Mariano Ospina Pérez del municipio de Tinjacá, durante el desarrollo de ejes temáticos 
en las áreas de química y matemáticas. Esto nos ha dejado ver que sí es posible disminuir la apatía por el estudio de estas dos áreas, y que el juego sí funciona como propuesta para lograrlo. En tanto que desde la construcción de cada uno de los juegos se mostraron aptitudes interesantes (como el trabajo en equipo) y se descubrieron talentos en estudiantes que aportaron a cada equipo que se conformó dentro del aula, para luego ver con alegría como fruto del trabajo en talleres con ese material, la concepción de un ambiente de armonía y comunicación no solo entre estudiantes sino con cada docente.

\section{Referencias}

Bausela, E. (2002). La docencia a través de la investigación-acción. Revista Iberoamericana de educación, 72(6). España: Universidad de león. Recuperado de: http://rieoei.org/profesion25.htm

Chacón, P. (2008). El juego didáctico como estrategia de enseñanza y aprendizaje: ¿Cómo crearlo en el aula? Nueva Aula Abierta, 5(16).

De Guzmán, M. (1984). Juegos matemáticos en la enseñanza. Actas de las IV Jornadas sobre Aprendizaje y Enseñanza de las Matemáticas Santa Cruz de Tenerife. Universidad complutense de Madrid.

Fernández, M. (2014). El juego y las matemáticas. (Trabajo de pregrado). Universidad de la Rioja, Logroño, España.

Gómez-Duarte, L., \& Peñaloza-Jiménez, G. (2014). Didáctica y comunicación de Habermas a la educación. Praxis \& Saber, 5(9), 13-29. https: // doi. org/10.19053/22160159.2991

Guapacha, G (2013). El juego como estrategia en la enseñanza-aprendizaje de la nomenclatura inorgánica. Manizales: Universidad Nacional de Colombia.

MEN (2015). Ruta de reflexión y mejoramiento pedagógico: "siempre día E". Colombia. Recuperado de http://aprende.colombiaaprende.edu.co/sites/ default/files/naspublic/Guia\%204_Ciclo\%203_V4ok.pdf

Muñiz, L., Alonso, P., \& Rodríguez, L. (2014). El uso de los juegos como recurso didáctico para la enseñanza y el aprendizaje de las matemáticas: Estudio de una experiencia innovadora. Revista iberoamericana de educación matemática, (39), 19-33.

Orrego, J., \& Toro, L. (2014). Relaciones vitales: el aula como escenario permanente de investigación. Praxis \& Saber, 5(10), 121-139. https: // doi. org/10.19053/22160159.3025 
Paredes, J. (2011). La relación pedagógica y la evaluación Jornada sobre relación pedagógica. Madrid, España: Universidad Autónoma de Madrid

Posada, R (2014). La lúdica como estrategia didáctica. (Trabajo de grado). Universidad Nacional de Colombia, Bogotá, Colombia.

Proyecto Educativo Institucional (2016). Documento actualizado PEI Institución Educativa Mariano Ospina Pérez. Tinjacá.

Rodríguez, E. (2013). El aprendizaje de la química de la vida cotidiana en la Educación básica. ARJÉ, revista de postgrado face-UC. 7(12), 363-373. Recuperado de http://servicio.bc.uc.edu.ve/educacion/arje/arj12/art21.pdf

Rodríguez, G. G., Gil, F. J., \& García, J. E. (1996). Metodología de la investigación cualitativa. Granada, España: Ediciones Aljibe.

Samacá, I. (2016). El espíritu científico en la primera infancia. Praxis \& Saber, 7(13), 89-106. https://doi.org/10.19053/22160159.4167

Teoría de los juegos. (2012). Teorías de los Juegos: Piaget, Vigotsky, Groos. Recuperado dehttps://actividadesludicas2012.wordpress.com/2012/11/12/teorias-de-losjuegos-piaget-vigotsky-kroos/

Vacca, R. (2012). Apatía hacia las matemáticas. Pasto, Colombia. Universidad ICESI.

Valverde, G., \& Näslund, E. (2010). La condición de la educación en matemáticas y ciencias naturales en América Latina y el Caribe. Banco Interamericano de Desarrollo. 